\title{
Anmerkungen zur Notation
}

Unter einem Phänomen/Sachverhalt wird - ausgehend von der Grundannahme Kants, dass uns nicht die „Dinge an sich“, sondern nur deren Erscheinungen zugänglich sind (vgl. Kant 1787/1966: 28ff. (= B XVI ff); vgl. auch Felder 2009b: 16), - mit Rückgriff auf das Zeichenmodell von Peirce (vgl. Kap. 2.2.2) und in Anlehnung an Köllers Interpretation desselben ein „Zeichenobjekt“ verstanden. Köller beschreibt das „Zeichenobjekt“ nach Peirce wie folgt:

Das Zeichenobjekt ist für Peirce eine Sachgröße, die mit Hilfe des jeweiligen Zeichens als Teilgröße aus dem Kontinuum der physischen oder der geistigen Welt herausdifferenziert wird und die deshalb als pragmatische Ursache der ganzen Zeichenbildung anzusehen ist.

(Köller 2004: 243)

Phänomene werden durch Kapitälchen (z. B. BuRNouT) notiert.

Die Unterscheidung zwischen Wort bzw. Ausdruck und Begriff/Konzept begründet sich in der linguistischen Unterscheidung zwischen Form- und Inhaltsseite bzw. „zwischen Gestalt und Wert“ (Bär 2015: 176). Unter Begriff/ Konzept wird in dieser Arbeit vor dem Hintergrund erkenntnistheoretischer Überlegungen zur Perspektivität (sprachlicher) Zeichen (Köller 2004) eine durch (sprachliche) Zeichen vermittelte Interpretationsperspektive auf ein Phänomen (vgl. das Zeichenmodell von Peirce, Kap. 2.2.2) bzw. einen Phänomenbereich verstanden, die besondere Aspekte an diesem Phänomen hervortreten lässt und das Phänomen auf besondere Weise in bestehendes Wissen/Denkhorizonte einordnet.

Die Arbeit verwendet die Ausdrücke Begriff und Konzept weitgehend synonym. Der Begriffsbegriff betont jedoch stärker die Abhängigkeit der Interpretationsperspektive von ausdrucksseitigen Objektivierungsformen und reflektiert das Vorgehen der interpretierenden Person dahingehend, dass verschiedene objektsprachliche Ausprägungen einer Interpretationsperspektive beschreibungssprachlich möglichst prägnant gefasst, sprich „auf den Begriff“ gebracht werden müssen (vgl. Bär 2015: 178; 183). Um bei Begriffen die Bedeutung der Ausdrucksund Inhaltsseite zu betonen, werden sie sowohl kursiv als auch mit romanischen einfachen Anführungszeichen (z. B. `Burnouts) gesetzt. Die romanischen einfachen Anführungszeichen werden in der Arbeit sonst für `Konzepte verwendet. Wenn die Konzept- bzw. Begriffs- und Sachverhaltsebene gleichermaßen aufgerufen werden soll, wird ebenfalls mit Mehrfachmarkierung gearbeitet: >BURNOUT<.

Die |Notation| mit senkrechten Strichen markiert Praktiken mit definitorischer Funktion (in den Kapiteln 6.2.2.4, 6.2.3.3 und 7.2). 
Uneigentlicher, distanzierender Sprachgebrauch wird durch einfache Anführungszeichen ,so' markiert und Teilbedeutungen werden 'so' angezeigt. Längere Zitate (mehr als drei Zeilen) werden durch Einrückung und eine um zwei Punkte kleinere Schriftgröße als der Haupttext angezeigt oder stehen merklich abgesetzt vor Kapitelbeginn. 